Article available at http://www.parasite-journal.org or http://dx.doi.org/10.1051/parasite/2001081061

\title{
Profil Séro-ÉPIdémiologiQue de la toxoplasmose AU NORD DE LA TUNISIE
}

\author{
BOURATBINE A.*, SIALA E.*, CHAHED M.K.**, AOUN K.* \& BEN ISMAIL R.*
}

Summary : SERO-EPIDEMIOLOGICAL DATA ABOUT TOXOPLASMOSIS IN THE NORTH OF TUNISIA

Toxoplasma antibodies prevalence was studied in the north of Tunisia where a mild climate prevails. Two groups of individuals were investigated: 857 living in rural area and 564 living in urb town. Sera were analysed by ELISA and indirect

immunofluorescence. The overall prevalence was $58.4 \%$. It roses from $24.5 \%$ at ten years to $52.1 \%$ at 20 years of age. A maximum level, around $70 \%$, was reached by about 30 years. The risk of acute infection after this age seemed low as judging by the proportion of high antibodies titers observed in this group $114.2 \%$ before 30 years vs $3.7 \%$ after). A significantly higher prevalence was detected in urban residents $167 \%$ vs $52.8 \%$ ). In this group, the rate of seroconversion seems the highest between ten and 20 years of age and the majority of women are infected before reaching childbearing age. In the rural area, the seropositivity is lower between ten-20 years and many women at childbearing age still suceptible to toxoplasmosis. The risk of acute infection seems higher in the youngest ones as showed by the proportion of high antibodies titers observed in the 18-30 age group $(9.2 \%$ ) compared to the one observed after 30 years (1.9\%).

KEY WORDS : toxoplasmosis, epidemiology, rural area, urbain area, Tunisia.

\section{INTRODUCTION}

L a toxoplasmose est une parasitose cosmopolite. Cependant, sa prévalence et son incidence varient dentre les populations, probablement en rapport avec une exposition différente aux multiples facteurs de risque (Dubey, 1988; Paris, 1996).

L'infection par T. gondii est particulièrement importante à considérer chez la femme enceinte, le parasite pouvant être transmis au foetus et lui occasionner des malformations graves (Wong, 1994). En Tunisie, malgré l'absence d'un programme national de prévention, la sérologie toxoplasmique est systématiquement demandée dans les grands centres hospitaliers dès la première consultation prénatale (Ben Ayed,

\footnotetext{
* Laboratoire de Parasitologie, Institut Pasteur de Tunis.

** Section de Médecine préventive, Faculté de Médecine de Tunis. Correspondance : Pr Bouratbine Aîda. Laboratoire de Parasitologie, Institut Pasteur de Tunis, 13, place Pasteur, BP 74, 1002 Tunis, Tunisie. Tél. : 002161792429 - Fax : 002161791833.
}

\begin{abstract}
Résumé :
La prévalence de la toxoplasmose a été étudiée dans le nord de la Tunisie chez 857 individus d'origine rurale et 564 individus d'origine urbaine. Les anticorps anti-toxoplasmiques ont été recherchés par immunofluorescence indirecte (IFI) et par ELISA. La prévalence de la toxoplasmose dans la population générale était de $58,4 \%$. Elle a significativement varié avec l'âge, passant de $24,5 \%$ à dix ans à $52,1 \%$ à 20 ans, pour atteindre un maximum autour de $70 \%$ vers l'âge de 30 ans. À partir de cet agge, les séroconversions semblent rares à en juger par la faible proportion des sérums à titres élevés en IFI 1 14,2\% akant 30 ans vs 3,7\% après 30 ans). La prévalence était significativement plus élevée en milieu urbain (67\% vs 52,8\%). Dans ce milieu, c'est entre dix et vingt ans, que les séroconversions semblent les plus fréquentes, la majorité des femmes étant alors immunisées à l'âge de la procréation. En milieu rural, l'immunisation, moins intense entre dix et 20 ans, fait que le nombre de femmes réceptives à la toxoplasmose à l'âge légal du mariage (18 ans) est plus important. Ce sont donc les femmes jeunes de ce milieu qui sont les plus exposées au risque d'infection toxoplasmique comme en témoigne la proportion élevée de sérum fortement positifs dans cette population $19,2 \%$ pour les $18-30$ ans vs $1,9 \%$ pour les 30 48 ans!.
\end{abstract}

MOTS CLÉS : toxoplasmose, épidémiologie, milieu rural, milieu urbain, Tunisie.

1994; Khémiri, 1997). Cependant, cette première sérologie est très souvent tardive, demandée au cours du deuxième trimestre (Khémiri, 1997). L'interprétation dès lors de certains résultats sérologiques devient délicate (Jenum, 1998).

L'institution d'un programme national de prévention, basé, tel que préconisé dans d'autres pays, sur la détermination du statut immunitaire de la femme en prénuptial et en prénatal (Wallon, 1994) permettrait de pallier à ces insuffisances. La pertinence de sa mise en place dépend cependant de l'amplitude du problème dans la communauté et du risque réel de la contamination de la femme parturiente (Desmont, 1990; Lappalainen, 1995).

Afin d'émettre des hypothèses quant aux modalités de transmission du parasite et d'apprécier le risque de séroconversion des femmes en âge de procréer selon leur mode de vie, une étude du profil séro-épidémiologique de la toxoplasmose a été réalisée dans deux communautés, l'une rurale et l'autre urbaine, du nord de la Tunisie. 


\section{MATÉRIEL ET MÉTHODES}

\section{LIEU DE L'ENQUÊTE}

T

a transmission de la toxoplasmose a été étudiée dans deux délégations limitrophes du gouverunorat de Béja (nord de la Tunisie) : la délégation de Medjez el Bab et la délégation de Goubellat. L'étude a concerné les populations de deux "imadats" : Khniguet Eddhen (KDH) et Medjez El Bab Ville (MBV). Cette zone géographique appartient à la région du Tell qui se caractérise par un climat de type méditerranéen semi-humide à sub-humide avec une température moyenne annuelle située entre $18^{\circ} \mathrm{C}$ et $20^{\circ} \mathrm{C}$ et une pluviométrie annuelle entre 400 et $600 \mathrm{~mm}$.

La première "imadat", $\mathrm{KDH}$, est une zone rurale qui compte environ 1400 individus. L'approvisionnement en eau s'y fait par des -moyens traditionnels (puits, citernes, fontaines publiques) et le métier prédominant y est celui d'ouvrier agricole. La deuxième "imadat", $\mathrm{MBV}$, est une zone urbaine où résident 1250 individus environ. L'approvisionnement en eau y est assuré par le réseau national de distribution des eaux et les métiers commerciaux et administratifs y sont prédominants.

\section{CARACTÉRISTIQUES DE LA POPULATION ÉTUDIÉE}

L'enquête sur le terrain a été réalisée en deux temps par l'équipe du Laboratoire d'Épidémiologie et d'Écologie parasitaire de l'Institut Pasteur de Tunis dans le cadre d'un projet sur l'hydatidose. D'abord, les populations des deux "imadats" ont été recensées lors de visites au porte-à-porte. Secondairement, des prélèvements de sang qui se voulaient exhaustifs ont été effectués. Les sérums ont été conservés à $-20^{\circ} \mathrm{C}$. Pour les besoins de notre étude, nous avons pu accéder à la sérothèque et aux données concernant l'âge, le sexe et l'état civil des individus recensés.

La population de KDH est formée de 1415 individus groupés en 262 familles vivants dans 166 habitations. La population est jeune, majoritairement constituée d'individus de moins de 30 ans (62\%). Les deux sexes sont représentés de manière équivalente. 55,3\% de la population féminine a entre 18 et 48 ans. Parmi cette population en âge de procréer, $56 \%$ ont moins de 30 ans. La proportion de femmes mariées diffère en fonction de la tranche d'âge (20\% des 18-30 ans et $80 \%$ des 30-48 ans). MBV compte 1256 individus groupés en 310 familles vivant dans 258 habitations. La population est moins jeune que celle de $\mathrm{KDH}$, la médiane d'âge étant de 30 ans. Comme à KDH, les sujets de sexe masculin et ceux de sexe féminin sont également répartis dans la population. La population féminine âgée de 18 à 48 ans représente 52,8\% de la totalité des individus de sexe féminin, $53 \%$ d'entre elles ont moins de 30 ans. La proportion des femmes mariées est également différente en fonction de l'âge (30\% des 18-30 ans et $87 \%$ des 30-48 ans).

Les sérums de 1421 individus ont été étudiés. 857 provenaient de $\mathrm{KDH}$ et 564 de $\mathrm{MBV}$. Ce qui représente $60,5 \%$ de la population rurale et $45 \%$ de la population urbaine. Les individus de sexe féminin étaient dans les deux cas plus prélevés que ceux de sexe masculin, respectivement $74 \%$ versus $53 \%$ à $\mathrm{KDH}$ et $50 \%$ versus $40 \%$ à MBV.

\section{SÉROLOGIE TOXOPLASMIQUE}

Les anticorps (Ac) anti-toxoplasmiques ont été recherchés et titrés systématiquement par deux techniques, l'immunofluorescence indirecte (IFI) et l'ELISA. L'antigène utilisé en IFI est commercialisé par l'Institut Pasteur de Tunis. La révélation des anticorps a utilisé une antiglobuline humaine dirigée contre les Ig GAM (Sanofi Diagnostics Pasteur). Les immunoglobulines totales ont été quantifiées en unités internationales par rapport à un étalon OMS. Le seuil de positivité de la technique est de $10 \mathrm{UI} / \mathrm{ml}$. Le kit utilisé en ELISA est commercialisé par Sanofi Diagnostic Pasteur (Platelia Toxo Ig G), son seuil de positivité est de 6 UI.

Tout sérum positif par au moins l'une de ces deux techniques a été rețenu comme positif. Un titre d'anticorps supérieur ou égal à $256 \mathrm{UI} / \mathrm{ml}$ en IFI a été considéré comme élevé.

\section{ANALYSE STATISTIQUE}

L'analyse statistique des données a été réalisée à l'aide du logiciel SPSS/Windows : version 7.0. Le test du Chi 2 et le test $t$ de Student ont été utilisés pour comparer respectivement les pourcentages et les moyennes.

\section{RÉSULTATS}

\section{RÉSULTATS SÉROLOGIQUES}

DE LA POPULATION GLOBALE

$\mathrm{P}$ armi les 1421 sérums testés, 831 soit $58,4 \%$ se sont révélés positifs. Le taux de concordance entre les deux techniques était de 98,8\%.

La séroprévalence a significativement varié en fonction de l'âge $(p<0,001)$ et du milieu $(p<0,001)$.

En fonction de l'âge, la séroprévalence a progressivement augmenté jusqu'à 30 ans, pour se stabiliser par la suite autour d'une valeur moyenne de $70 \%$. À partir de cet âge, les sérums à titre élevé d'Ac étaient plus rares $(3,7 \%$ après 30 ans versus $14,2 \%$ avant 30 ans; $p<0,001$ ) (Tableau I , Fig. 1).

La séroprévalence était plus élevée en milieu urbain qu'en milieu rural (67\% versus 52,8 \%). Cependant, cette comparaison globale n'a pas de valeur statistique 


\begin{tabular}{rrrrrr}
\hline & Négatifs & Positifs & Total & $\begin{array}{c}\text { Séro- } \\
\text { prévalence }\end{array}$ & $\begin{array}{c}\text { IFI > 256 UI } \\
\text { (\% dans la } \\
\text { population) }\end{array}$ \\
\hline $\mathbf{1 - 1 0}$ ans & 166 & 54 & 220 & $24,5 \%$ & $45(20,5 \%)$ \\
$\mathbf{1 0 - 2 0}$ ans & 159 & 173 & 332 & $52,1 \%$ & $52(15,7 \%)$ \\
$\mathbf{2 0 - 3 0}$ ans & 98 & 178 & 276 & $64,5 \%$ & $21(7,6 \%)$ \\
$>\mathbf{3 0}$ ans & 167 & 426 & 593 & $71,8 \%$ & $22(3,7 \%)$ \\
Total & 590 & 831 & 1421 & $58,4 \%$ & $140(9,8 \%)$ \\
\hline
\end{tabular}

Tableau I. - Séroprévalence et proportion de sérums à titre élevé en fonction de l'âge dans la population d'étude.

sans une analyse des effectifs par classe d'âge. En effet, la classe un-dix ans représente $17 \%$ des sérums en milieu rural et $13 \%$ en milieu urbain. La classe dix20 ans : $43 \%$ en milieu rural et $33 \%$ en milieu urbain. La séroprévalence étant plus faible dans ces classes d'âge, leur sur-représentation en milieu rural par rapport au milieu urbain peut à elle seule expliquer la différence significative entre les deux groupes lorsque l'on compare l'ensemble des résultats. L'analyse comparative des séroprévalences par tranche d'âge dans les deux milieux a cependant montré que, si avant dix ans la prévalence n'était pas significativement différente (29,3\% en milieu urbain et $22,1 \%$ en milieu rural), elle l'était par contre entre dix et 20 ans $(67 \%$ en milieu urbain versus $44,8 \%$ en milieu rural, $p<0,001$ ) et se maintenait au-delà de 20 ans $(74,4 \%$ versus $65,6 \%, p$ $<0,05$ ) (Tableau II, Fig. 2). En ce qui concerne les sujets porteurs de titres élevés d'Ac antitoxoplasmiques, il n'a pas été noté de différence significative entre les tranches d'âges des deux milieux (Tableau II).

\section{RÉSULTATS SÉROLOGIQUES}

DE LA POPUlation FÉMININE EN ÂGE DE PROCRÉER

Chez les 388 individus de sexe féminin âgés de 18 à 48 ans, $67 \%$ avaient des anticorps antitoxoplasmiques. $33 \%$ étaient donc réceptives à la toxoplasmose. 4,9\% de cette population avait un titre élevé d'Ac antitoxoplasmiques.

La séroprévalence n'était pas significativement différente entre les tranches d'âge ou entre les deux milieux rural et urbain (Tableaux III et IV).

Les sérums fortement positifs sont trouvés en proportion plus élevée chez les femmes jeunes de 18-30 ans ( $6,8 \%$ versus $2,7 \%$, Tableau III). Cependant, cette différence n'est significative que chez les femmes d'origine rurale $(9,2 \%$ versus $1,9 \%, p<0,05$, Tableau IV). Ces femmes rurales de moins de 30 ans sont à $80 \%$ des célibataires.

\begin{tabular}{|c|c|c|c|c|c|c|c|c|c|c|}
\hline & \multicolumn{5}{|c|}{ Milieu rural } & \multicolumn{5}{|c|}{ Milieu urbain } \\
\hline & - & + & Total & Prévalence & $>256 \mathrm{U}(\%)$ & - & + & Total & Prévalence & $>256 \mathrm{U}(\%)$ \\
\hline $1-10$ & 113 & 32 & 145 & $22,1 \%$ & $28(19,3 \%)$ & 53 & 22 & 75 & $29,3 \%$ & $17(22,7 \%)$ \\
\hline $10-20$ & 123 & 100 & 223 & $44,8 \%{ }^{1}$ & $31(13,9 \%)$ & 36 & 73 & 109 & $67 \% 1$ & $21(19,3 \%)$ \\
\hline $20-30$ & 64 & 108 & 172 & $62,8 \%$ & $16(9,3 \%)$ & 34 & 70 & 104 & $67,3 \%$ & $5(4,8 \%)$ \\
\hline$>30$ & 104 & 213 & 317 & $67,2 \%{ }^{2}$ & $11(3,5 \%)$ & 63 & 213 & 276 & $77,2 \%{ }^{2}$ & $11(4 \%)$ \\
\hline Total & 404 & 453 & 857 & $52,8 \%$ & $86(10 \%)$ & 186 & 378 & 564 & $67 \%$ & $54(9,5 \%)$ \\
\hline
\end{tabular}

$1: p<0,001 ; 2: p<0,05$.

Tableau II. - Séroprévalence et proportions de sérums fortement positifs en fonction de l'âge, en milieu rural et urbain.

Fig. 1. - Séroprévalence dans la population générale.

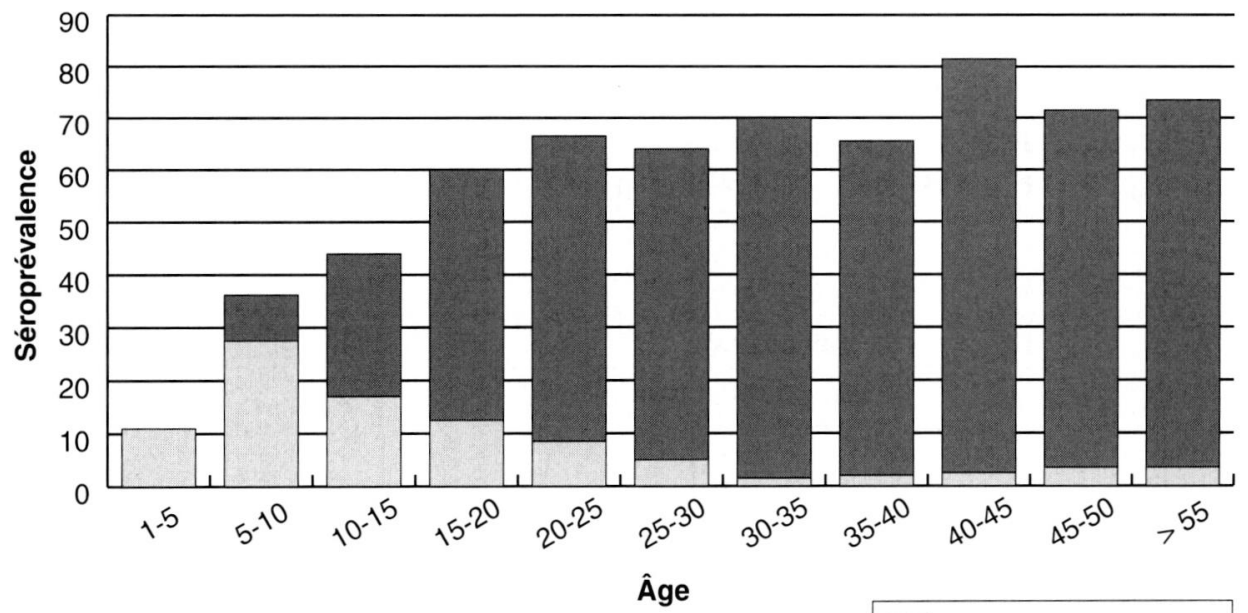

$\%$ de sérums à titre éleve 


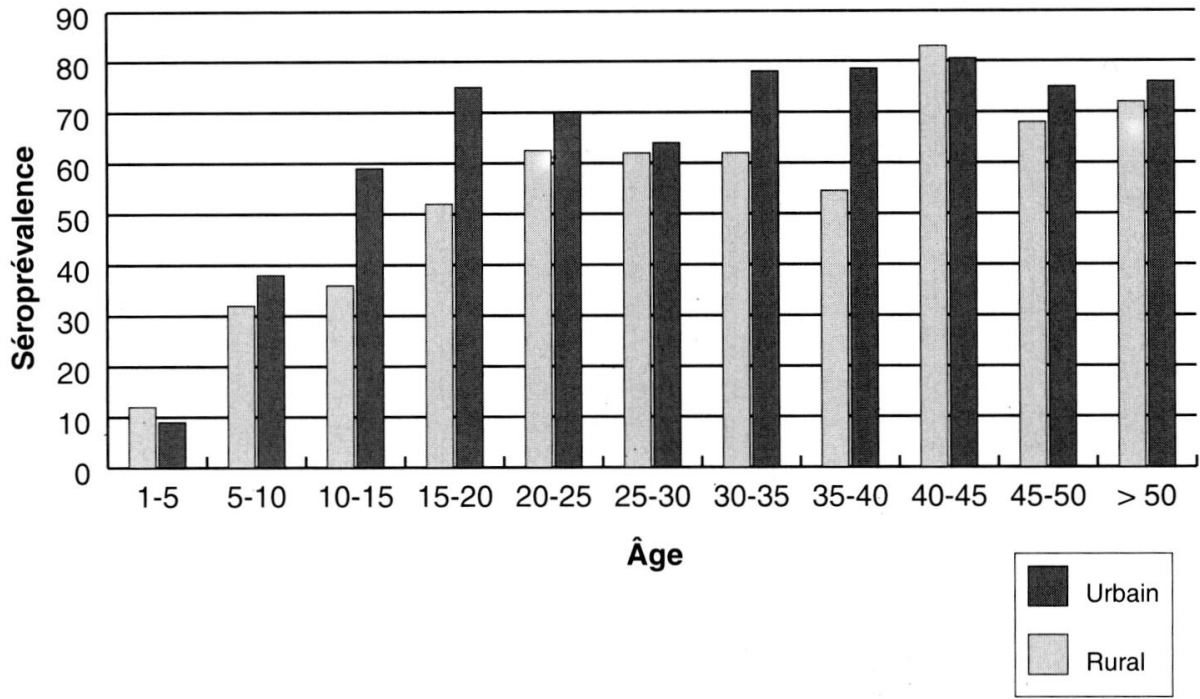

Fig. 2. - Séroprévalence en milieux rural et urbain.

\begin{tabular}{lccccc}
\hline & & & & $\begin{array}{c}\text { IFI }>\mathbf{2 5 6} \text { UI } \\
\text { Séro- }\end{array}$ \\
Négatifs & Positifs & Total & $\begin{array}{c}\text { prévalence } \\
\text { de sujets) }\end{array}$ \\
\hline $\begin{array}{l}\text { 18-30 ans } \\
\text { 30-48 ans }\end{array}$ & 69 & 136 & 205 & $66,3 \%$ & $14(6,8 \%)$ \\
Total & 128 & 124 & 183 & $67,6 \%$ & $\begin{array}{r}5(2,7 \%) \\
19(4,9 \%)\end{array}$ \\
\hline
\end{tabular}

Tableau III. - Séroprévalence et proportion de sérums fortement positifs chez les femmes en âge de procréer.

\section{DISCUSSION}

L a séroprévalence de la toxoplasmose est de l'ordre de $70 \%$ chez les adultes de la région étudiée. Ce chiffre se rapproche de ceux observés dans les zones cotières et humides d'Afrique du Nord (DupouyCamet, 1993; Biava, 1983). Il reflète probablement un niveau de transmission élevé dans le nord du pays où le degré élevé d'hygrométrie est favorable au maintien des oocystes (Dupouy-Camet, 1993). D'autres études effectuées dans d'autres régions à climat plus sec (centre et sud) pourraient révéler, à l'instar d'autres pays maghrebins, des séroprévalences plus basses (Biava, 1983). L'évaluation de la séroprévalence en fonction de l'âge est d'un grand intérêt dans la détermination du profil épidémiologique de la toxoplasmose dans une région donnée (Walker, 1992; Carme, 1996; Dupouy-Camet, 1993). Ainsi, il apparaît dans la population étudiée que, entre un et dix ans, la proportion d'enfants immunisés est déjà élevée (24,5\%). Entre dix et 20 ans, l'immunisation continue de progresser. À partir de 20-30 ans, le niveau d'immunisation maximum est atteint. Plus de $70 \%$ des sujets sont alors porteurs d'Ac antitoxoplasmiques et les infections deviennent rares. Ce profil épidémiologique a déjà été décrit par d'autres auteurs tunisiens (Messedi-Triki, 1982). Il est en faveur d'une contamination précoce, se faisant dans l'enfance et l'adolescence. Comme dans les zones tropicales, durant cette période de vie, la transmission par les oocystes est probablement prépondérante. À coté de ce mode de transmission, coexisteraient des contaminations itératives par les kystes contenus dans la viande d'ąnimaux de boucherie. Cette modalité de contamination expliquerait ainsi, que, entre dix et 30 ans, alors que l'hygiène s'améliore et le contact tellurique diminue, les individus continuent à s'immuniser. Il est à signaler que dans la région de l'étude, le mouton constitue l'animal de boucherie le plus commun. Ce dernier est en Tunisie celui qui est trouvé le plus infecté par Toxoplasma gondii, avec des taux de positivité avoisinant les 75 \% (Ben Rachid, 1970). La consommation de sa

\begin{tabular}{|c|c|c|c|c|c|c|c|c|c|c|}
\hline & \multicolumn{5}{|c|}{ Milieu rural } & \multicolumn{5}{|c|}{ Milieu urbain } \\
\hline & - & + & Total & Prévalence & $>256 \mathrm{U}(\%)$ & - & + & Total & Prévalence & $>256 \mathrm{U}(\%)$ \\
\hline $18-30$ & 42 & 89 & 131 & $67,9 \%$ & $12(9,2 \%)^{1}$ & 27 & 47 & 74 & $63,5 \%$ & $2(2,7 \%)$ \\
\hline $30-48$ & 37 & 67 & 104 & $64,4 \%$ & $2(1,9 \%)^{1}$ & 22 & 57 & 79 & $72,2 \%$ & $3(3,8 \%)$ \\
\hline Total & 79 & 156 & 235 & $66,4 \%$ & $14(6 \%)$ & 49 & 104 & 153 & $68 \%$ & $5(3.3 \%)$ \\
\hline
\end{tabular}

$1: p<0,05$.

Tableau IV. - Séroprévalence et proportion de sérums fortement positifs chez les femmes en âge de procréer, en milieu rural et urbain. 
viande grillée (méchoui) est fréquente, surtout lors de certaines fêtes religieuses "zerda" ou de la cérémonie annuelle du sacrifice (Aïd El Kebir).

La séroprévalence de la toxoplasmose est significativement plus élevée en milieu urbain qu'en milieu rural $(p<0,001)$. En Tunisie, la plupart des études antérieures n'ont concerné que des populations urbaines. Ailleurs, dans d'autres régions du monde, une telle différence n'a été signalée qu'en République du Congo (Dumas, 1990).

Cette différence de prévalence ne semble pas liée à l'âge de la population étudiée, globalement plus jeune en milieu rural. Elle se retrouve en effet dans toutes les tranches d'âge à partir de dix ans. Il est très probable que ce niveau plus élevé en milieu urbain reflète une contamination par consommation plus fréquente de viande ovine. L'importance d'une telle consommation dépendant des habitudes culinaires mais surtout du niveau socio-économique. Il est également possible que ce niveau plus élevé soit la conséquence d'un réservoir félin plus important en ville. La densité des chats, leur structure en âge et leur potentiel reproductif étant déterminants dans la transmission en zone urbaine (Sousa, 1988). L'existence dans ce milieu des mouches et des blattes serait un facteur de risque supplémentaire. Ils agiraient comme transporteurs des oocystes vers l'alimentation (Frenkel, 1995). L'importance relative des différentes modalités de contamination dans les deux milieux ne pourrait en fait être définie que par une exploration des facteurs de risque. Dans l'avenir, la caractérisation d'antigènes spécifiques de stades permettra de différencier par une simple réaction sérologique, entre la contamination par les kystes et celle par les oocystes (Kasper, 1989).

Les femmes en période de procréation constituent le groupe à risque le plus important, en raison de la gravité des foetopathies pouvant résulter d'une infection toxoplasmique acquise au cours d'une grossesse (Wong, 1994). Les résultats de notre enquête montrent que $66,9 \%$ des femmes en âge de procréer possèdent une immunité toxoplasmique résiduelle protectrice qui les met à l'abri d'une toxoplasmose évolutive. Ce taux est proche de celui rapporté chez les parturientes consultant dans divers hôpitaux de la région de Tunis (Ben Ayed-Nouira, 1994; Khémiri, 1997). La proportion de femmes porteuses d'un titre élevé d'Ac (4,7\%) laisse cependant présager que, dans ce groupe à risque, les infections ne sont pas exceptionnelles. En fait, c'est entre 18 et 30 ans que ces titres élevés, probablement témoins d'infections récentes (Candolfi, 1990), sont les plus fréquemment rencontrés. Dans notre population, ils ont essentiellement été trouvés chez les femmes d'origine rurale. Ceci peut s'expliquer par un nombre de sujets réceptifs à l'âge de 18 ans plus important dans ce milieu. En milieu urbain, à l'âge légal du mariage (18 ans), comme déjà rapporté par d'autres auteurs tunisiens (Jemni, 1986), la plupart des femmes sont immunisées, le nombre de femmes ayant une sérologie fortement positive est bas et le risque d'infection semble faible.

Il est à noter que la contamination des femmes rurales, dans la tranche d'âges 18-30 ans peut être favorisée par l'hygiène précaire et le manque d'eau lors de la manipulation de la viande ovine pour la préparation des repas.

\section{CONCLUSION}

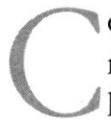

e travail a permis de préciser le profil épidémiologique de la toxoplasmose dans le nord de la Tunisie, dans deux milieux, l'un rural et l'autre urbain, et d'apprécier le risque encouru par les femmes en âge de procréer dans ces deux milieux. Toutefois, devant les variations régionales déjà mentionnées dans d'autres pays maghrébins, il paraît intéressant d'élargir ces enquêtes à différentes régions du centre et du sud tunisien à climat semi-aride ou aride où le degré d'hygrométrie est moins favorable à la transmission. Il est également intéressant d'étudier les profils sérologiques dans les grandes villes où l'environnement et le mode de vie de la population sont différents. Toutes ces études permettraient d'évaluer le risque réel de la toxoplasmose en Tunisie, de mesurer l'amplitude du problème dans la communauté tunisienne et d'évaluer la pertinence de l'institution d'un programme national de prévention de la toxoplasmose congénitale basé, tel que préconisé par d'autres pays, sur la détermination du statut immunitaire des femmes en prénuptial et/ou en prénatal.

\section{RÉFÉRENCES}

Ben Ayed Nouira N., Hafsia S., Khaled S. et al. Incidence de la toxoplasmose pendant la grossesse et risque de l'infection foetale. La Tunisie Médicale, 1994, 72 (8-9), 487-491.

Ben RACHID M.S. \& BlaHA R. La toxoplasmose humaine et animale en Tunisie. La Tunisie Médicale, 1970, 48 (2), 101-110.

Biava M.F., Jana M., El Mansouri A. \& Percebois G. Étude séroépidémiologique de la toxoplasmose à Marrakech (Maroc). Médecine et Maladies Infectieuses, 1983, 13, 503506

Candolfi E. \& Kien T. Les nouvelles données de l'interprétation de la sérologie de la toxoplasmose par l'évaluation comparée d'anciennes et de nouvelles techniques sérologiques. Spectra Biologie, 1990, 90 (1), 55-62.

Carme B. \& Tirard-Fleury V. La toxoplasmose chez la femme enceinte en France : séroprévalence, taux de séroconversion et niveau de connaissance des mesures préventives. Médecine et Maladies Infectieuses, 1996, 26, 431-436. 
Desmont G. Preventing congenital toxoplasmosis. Lancet, 1990, 336, 1017-1018.

Dubey J.P. \& BeATTIE C.P. Toxoplasmosis in animals and man. CRP Press, Boca Raton (Florida), 1988, 41-60.

Dumas N., Gazaux M., Carme B. \& Seguela J.P. La toxoplasmose en République du Congo. Étude séro-épidémiologique. Bulletin de la Societé de Pathologie Exotique, 1990, 83, 349-359.

Dupouy-Camet J., Gavinet M.F., Paugam A. \& Tourte SchaeFER Cl. Mode de contamination, incidence et prévalence de la toxoplasmose. Médecine et Maladies Infectieuses, 1993, 23, 139-147.

Frenkel J.K., Hassanein K.M., Hassanein R.S., Bwow E., Thulliez P. \& Quintero-Nunez R. Transmission of Toxoplasma gondii in Panama City, Panama. A five year prospective cohort study of children. American Journal of Tropical Medicine and Hygiene, 1995, 53, 458-468.

Jemni L., Bchir A., Faurant C., Heyer F., Braham M.S., AyaChi S., Djaidane A., Bouzakoura C. \& Lapierre I. La toxoplasmose et la rubéole à Sousse : une étude séroépidémiologique en milieu scolaire. La Tunisie Médicale, 1986, 64, 8-9.

Jenum P.A. \& Stray-Pedersen B. Development of specific immunoglobulins G, M and A following primary Toxoplasma gondii infection in pregnant women. Journal of clinical microbiology, 1998, 10, 2907-2913.

KASPER L.H. Identification of stage-specific antigens of Toxoplasma gondii. Infection and Immunity, 1989, 57, 668-672.

Khemiri B., Mahjoub S., Ben Hmid R., Lebbi I., Abed A., Sfar E., Ben Salem N., Zribi A. \& Chelli H. La séroprévalence de la toxoplasmose et de la rubéole parmi une population de femmes enceintes consultant au CMNRT : Service A. La Tunisie Médicale, 1997, 75, 788-793.

lappalainen M., Sintonen H., Koskiniemi M., Hedman K., Hillesmaa V., Ammala P., Teramo K. \& Koskela P. Cost benefit analysis of screening for toxoplasmosis during pregnancy. Scandinavian Journal of Infectious Diseases, 1995, 27, 265-272.

Messedi-Triki S., Memmi A. \& Ben Rachid M.S. Aspects épidémiologiques de la toxoplasmose en Tunisie. Le pharmacien du Maghreb, 1982, 2, 105-108.

Paris L. in: Traité de Parasitologie médicale. Édition Pradel, Paris, 1996.

Sousa O.E., Saenz R.E. \& Frenkel J.K. Toxoplasmosis in Panama: a 10 year-study. American Journal of Tropical Medicine and Hygiene, 1988, 38, 315-322.

WALKER J., NOKES D.J. \& JENnings R. Longitudinal study of Toxoplasma seroprevalence in south Yorkshire. Epidemiology and Infection, 1992, 108, 99-106.

Wallon M., Mallaret M.R., Mojon M. \& Peyron F. Toxoplasmose congénitale. Évaluation de la politique de prévention. Presse Médicale, 1994, 23, 1467-1470.

Wong S.Y. \& Remington J.S. Toxoplasmosis in pregnancy. Clinical infectious diseases, 1994, 18, 853-862.

Reçu le 25 mai 2000

Accepté le 24 octobre 2000 